\title{
Applicability of Table Reasoning Method for Fabric Defect Evaluation
}

\author{
By Cong Shao and Eiichi Kuze \\ Faculty of Science and Engineering, Science University of Tokyo, \\ Yamazaki Higashikameyama, Noda City, Chiba Prefecture, Japan \\ Based on the Journal of the Textile Machinery Society of Japan, Transactions, \\ Vol.42, No.3, T36-T46 (1989)
}

\begin{abstract}
Fabric inspectors can recognize the fabric defects because they can observe not only the light and darkness but also the brilliance or gloss on the fabric surface, So, in order to distinguish the fabric defects by machine, it is also necessary to deal with such information as gathered by men. In this paper, a new method was researched to distinguish the fabric defects. We shined laser light on fabric surface and measured the diffused light over 360 degrees to create a reflected pattern.

There are many naps and twists on the fabric surface irregularly, so the distribution of diffused light will be changed when the incident angle of laser is changed. If the light is shined on a fabric surface at an appropriate angle to detect the signal, the information about the fabric defect could be taken at its maximum. In our experiment, the most appropriate angle of incidence is 25 degrees and the most appropriate angle of measurement is 75 degrees.

By using a pinhole and a filter in the optical path, we normally came to be able to detect the signal even if not in drakroom. And when the pinhole diameter was $0.7 \mathrm{~mm}$, we detected the signal with averaging the rippl of the noise of the thread naps and twists, and without losing the defect signal. Here the measured area $(S)$ is given by the following equation.

$S=\pi a^{2} / 4 \cos \theta$

Where a is the pinhole diameter, and $\theta$ is the angle of measurement.

In this experiment, we found that the reflected pattern was dependent on the fabric construction. The normal pattern was symmetric, while abnormal one was distorted and the distortion of the reflected pattern was dependent on the kind of defect. The similar reflected patterns can be detected in the same sort of defect, but the identical one practically does not appear again even in the same sort of defect.

Table reasoning method was introduced in order to recognize the fabric defect with reflected pattern. Firstly twelve characteristic parameters were smapled from the reflected pattern to investigate the relations between reflected pattern and fabric defect, then an expert system was built to identify the fabric defects.
\end{abstract}

\section{Introduction}

The fabric has a very complicated three-dimensional structure. Moreover, the state of surface is also very complex. That is because not only there are many naps and roughness on the fabric surface but also the distribution is not so regular.

So the practical use of the automatic machine for detecting fabric defects is yet difficult, and the fabric inspection is also relyed on the hand. But, if a man works for a long time, he will get tired and will not be able to detect the defects accurately. Therefore, an automatic defect inspecting machine is expected to be realized.

The visual inspection by the naked eye usually depends on the strength of reflected light, color, and its distribution from fabric surface. That is to say, firstly the light is shined on the fabric surface, then the reflected light by eye is detected, and then this information is treated to judge by the inspector if there is a defect or not, or what kind of defect it is. If a machine also can collect the same optical information as a man, an automatic inspection also will be realized.

From this way of thinking, some basic research, for example, optical-electric method by using the reflected light $^{[1]}$, Fraunhofer diffraction method ${ }^{[2]}$, method by using both of reflection and transmitted light ${ }^{[3]}$, etc. have been reported. In these papers, because pieces of information treated were simpler than that by man, so even if the existence of defect could be detected, the king of defect couldn't be distinguished.

Detecting the existence of defect as well as the kind of defect will be required in order to realize the automatic 
defect inspection. In this paper, based on the besic research results of the previous researchers, the reflected pattern method was devised, which can gather more pieces of information than usual method. Moreover, table reasoning method was introduced to recognize the defect accurately by using the reflected pattern. Here we will report the composition of the signal detection, its foundamental performance, pattern creation, parameter calculation, dummy variable calculation, construction of table, and the defect distinguishment.

\section{Principle of Experiment}

The fabric inspector can recognize the fabric defects because they can observe not only the light and darkness but also the brilliance, or gloss of the fabric surface. Based on this way, we shined the laser on the fabric defect, and measured the reflected light around the defect over 360 degrees, then created a reflection pattern. And table resoning method was introduced to recognize the reflected pattern, in order to distinguish the fabric defect, because each reflected pattern was dependent on its fabric defect.

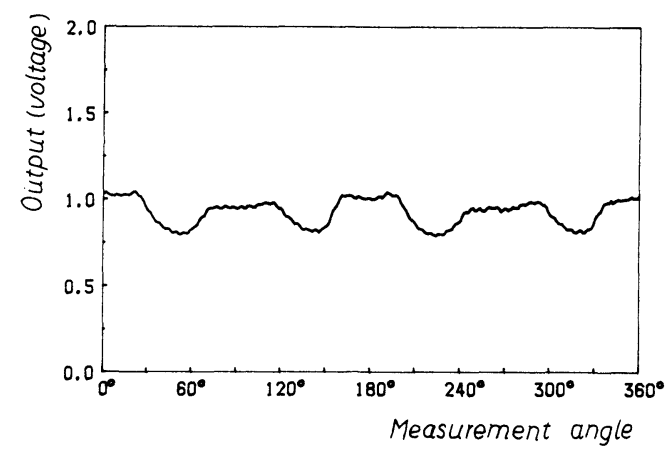

Fig. 1 The defect signal detected by PMT extending over $360^{\circ}$ at the normal part of the glass fiber fabric

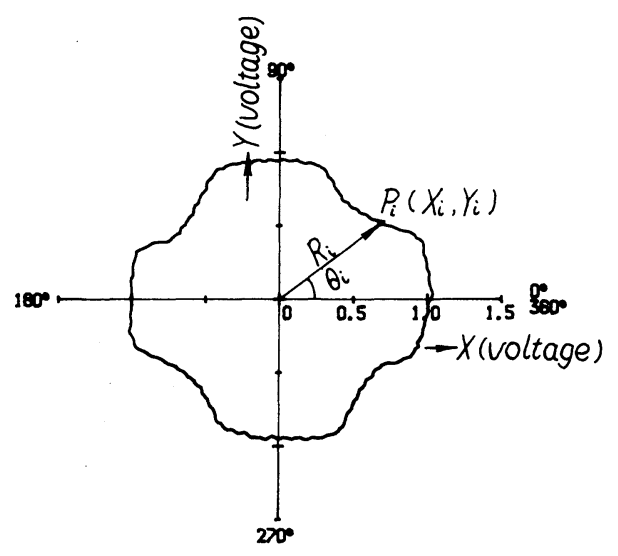

Fig. 2 The reflected pattern after coordinate transformation from the rectangular coordinate to the polar coordinate

\subsection{Pattern creation}

If we measure the reflected light over 360 degrees with rotating the rotational table, the signal shown in Fig. 1 will be obtained. After coordinate transformation from rectangular to polar coordinate, the signal shown in Fig. 1 becomes a closed curve shown in Fig. 2. Here, we call it as reflected pattern. From many experimental results, we found that very similar reflected patterns can be taken from the same kind of fabric defect, although they were not identical.

\subsection{Parameter calculation}

In order to recognize a closed curve such as the reflected pattern, for example a machine part as shown in Fig. 3, G.J.Agin ${ }^{[4]}$ firstly calculated the gravity center

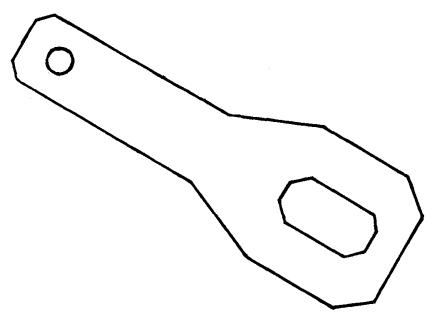

Fig. 3 An external form of machine parts

of the picture then calculated 7 characteristics $\left(X_{1}\right.$ : circumference length, $X_{2}$ : square root of area, $X_{3}$ : total area of hole, $X_{4}$ : minimum distance between the center of gravity and the outside, $X_{5}$ : maximum distance between the center of gravity and the outside, $X_{6}$ : average distance between the center of gravity and the outside, $X_{7}$ : slenderness). He reported that seven patterns could be discriminated by using these seven parameters shown in Fig. 4. In this case, the treated objects were machine parts, so the patterns taken from the same kind of machine parts were almost the same ones. Therefore the recognition of the existence was easily realized. In case of fabric, because defects were as many as scores of its kinds, and irregular roughness caused by the naps on fabric surface, the identical reflected pattern practically did not appear again even if from the same sort of fabric defect. It was clear that we should have increased the number of parameters in order to recognize such fuzzy patterns. Here twelve parameters were sampled from the reflected pattern to characterize the pattern. The twelve parameters such as $L$ (circumference length), $L_{s}$ (long and slender value), $D_{p}$ (distance between center of gravity and pattern center), $D_{i}$ (the shortest distance between center of gravity and outline), $D_{a}$ (the longest distance between center of gravity and outline), $I_{0}$ (moment of inertia), $R_{a}$ (aspect ratio), $R_{v}$ (average radius), $S_{n}$ (standard deviation), $S_{h}$ (average change ratio), $H_{d}$ (cave ratio), $J_{m}$ (repletion ratio) were calculated to be the characteristics data of reflected pattern. Where assuming the unit area density is 1 , and the pattern is as a plane. Assuming the radius $R_{i}$ is the distance between the center and 


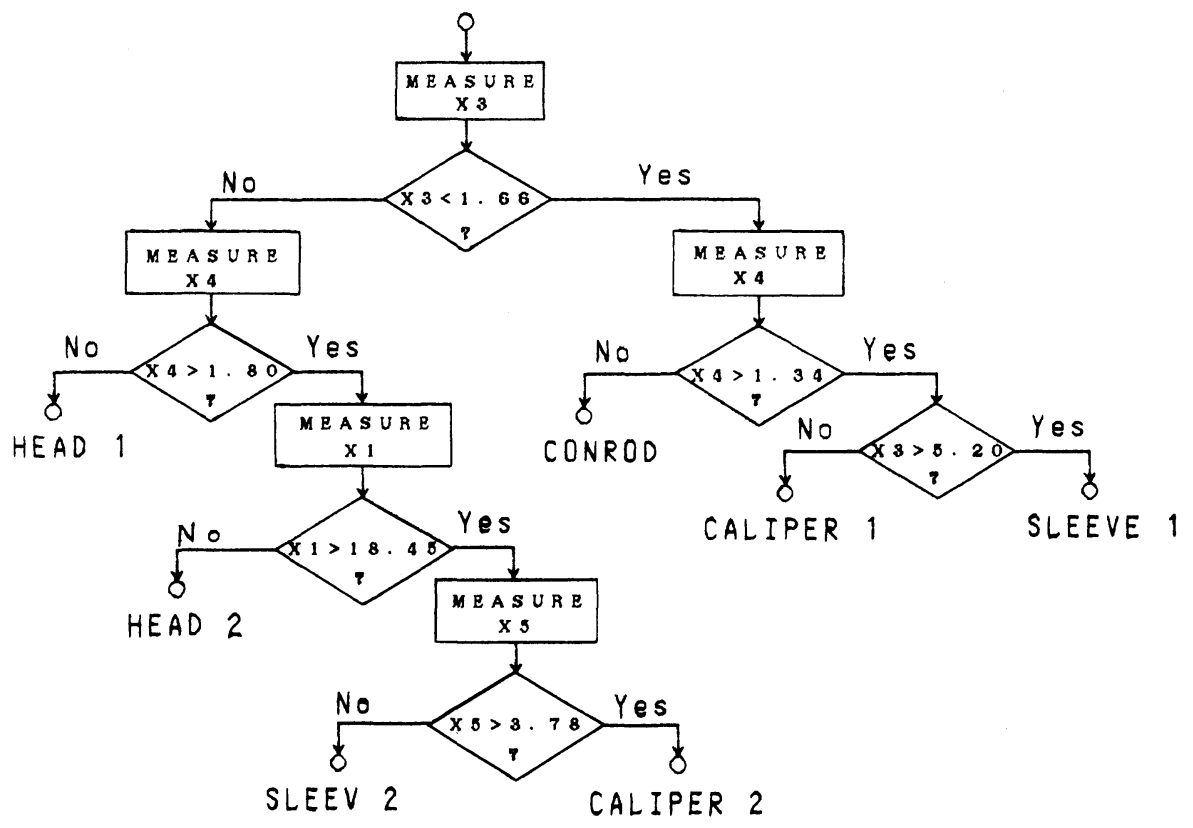

Fig. 4 G.J.Agin's distinguishing method by using seven characteristics

some point $P_{i}\left(X_{i}, Y_{i}\right)$ in the closed curve as shown in Fig. $2, L_{s}, R_{a}, S_{h}, H_{d}, J_{m}$ can be calculated by following equations.

$$
\begin{aligned}
L s & =L / S \quad \cdots \cdots \\
R_{a} & =\frac{Y \max -Y \min }{X \max -X \min } \cdots \\
B_{i} & =\frac{\Delta R_{i}}{\Delta \theta_{i}}=\frac{R_{i}+1-R_{i}}{2 \pi / n} \\
S_{h} & =\sum B_{i}{ }^{2} / n \\
& =\frac{n}{2 \pi}\left(\sum R_{i}-\sum R_{i} R_{i}+1\right) \\
H_{d} & =\frac{S \max -S}{S}=\frac{\pi R^{2} \max -S}{S} \\
J_{m} & =\frac{S-S \min }{S}=\frac{S-\pi R^{2} \min }{S}
\end{aligned}
$$

Where $S$ is the area of pattern, $n$ is the sampling numbers, $R_{\max }$ is the maximum radius, $S_{\max }$ is the maximum area, $R_{\min }$ is the minimum radius, $S_{\min }$ is the minimum area.

In order to reason defect by expert system with these parameters, dummy variables were introduced. We create the upper and lower limit of every parameter, and decide that if the calculated parameter is larger than the upper limit the dummy variable is 1 , if less than the lower limit the dummy variable is 3 , otherwise the dummy is 2 . These limits are shown in Table 1, and they can not be determined with trial and error until all defects are separated.
Table 1 Upper and lower threshold values (U.T. and L.T.)

\begin{tabular}{c|c|c}
\hline Parameter & U.T. & L.T. \\
\hline$L$ & 7.4865 & 6.8556 \\
\hline$L_{s}$ & 2.2207 & 1.9915 \\
\hline$D_{p}$ & 0.1079 & 0.0244 \\
\hline$D_{i}$ & 0.9505 & 0.8310 \\
\hline$D_{a}$ & 1.3297 & 1.1809 \\
\hline$I_{0}$ & 2.3026 & 1.6463 \\
\hline$R_{a}$ & 1.2775 & 1.0142 \\
\hline$H$ & 1.1024 & 0.9917 \\
\hline$S_{n}$ & 0.0197 & 0.0059 \\
\hline$S_{h}$ & 0.2864 & 0.1791 \\
\hline$H_{d}$ & 0.8261 & 0.3604 \\
\hline$J_{n}$ & 0.3869 & 0.2264 \\
\hline
\end{tabular}

\subsection{Reasoning table}

The fabric surface is very irregular, so the reflected pattern is fuzzy. Here, table reasoning method was introduced into the expert system to process such fuzzy reflected pattern.

Usually there are production rule, frame, etc. as knowledge management. The former is explained by using a simple explanation (If ... then ... ), so understanding, adding and amending will become easy. But the relation between the rules is not so obvious, the total image of the knowledge is hard to catch and the proces- 
sing speed is slow. On the other side, the frame has more flexibility and it is more useful, but, it is comparatively difficult to systematize a object.

On the other hand, the reasoning table ${ }^{[5]}$ that developed by Iwasaki Industry Company can easily represent a large quantity of knowledge. Referring some important portion of that manual, we explain the structure and the reasoning table at the next section.

\subsubsection{Construction of reasoning table}

Reasoning table is a method of approximate reasoning method. The reasoning table was constructed by head which becomes the candidacy for the conclusion or the interim hypothesis, weight coefficients (a contributing degree), and the threshold values. When reasoning, we compare the facts of the table with the truth facts. If the sum of the weights which exists in the inputted facts is over the threshold, the head of the table will be adopted as a conclusion or a interim hypothesis.

The status of the table is as follows.

Table name (Head, [Threshold, [Fact name 1, Fact value 1, weight 1], [Fact name 2, Fact value 2, weight 2], $\ldots \ldots \ldots$ [Fact name $n$, Fact value $n$, weight $n$ ] ] )

Where the table name is the name for recognizing the knowledge, here it is the identification of the fabric defect. The head is a candidacy of the conclusion, a defect name to reason. The fact name is a fact of an element to be selected as a candidacy for the conclusion. It can be obtained by asking or by reasoning, here it is one of that twelve parameters. The fact value is the value for comparing with that of the inputted fact, here it is a dummy variable. The weight is a contribution degree to be selected as conclusion for the candidacy. The threshold is a value to determine whether the result in the head will be or not be adopted when the total of the weights corresponding to the inputted fact is larger or less than the threshold.

The weight is experimentally decided with reference to the variation coefficient of each parameter. Firstly, each parameter is calculated, then the variation coefficient can be taken. If the variation coefficient is too large, it means that this parameter is not dependent so much on the defect comparing with the others. In this case, we will give weight small, or do not put it in the table (That is to say, make the weight to zero). On the contrary, if the variation coefficient is small, the depending degree of parameter will be large and make the weight large. But we can't decide the weight only by the variation coefficients.

\subsubsection{Reasoning method}

Now, supposing to reason some fabric defect (It is called as conclusion $\mathrm{A}$ ), if all the terms (parameters) for recognizing a defect is (1) as follows, following weights (2) to (1) are applied due to each term of experiment result.

$$
\left[a_{1}, a_{2}, a_{3}, \cdots \cdots a_{12}\right] \ldots \ldots \ldots \ldots
$$

$$
\left[\alpha_{1}, \alpha_{2}, \alpha_{3}, \cdots \cdots \alpha_{12}\right] \ldots \ldots \ldots \ldots
$$

These weights represent how much the fact terms contribute to the reason to adopt the conclusion $A$. If a reasoning with some parameters (3) (a fact group) taken from experiment is performed, and such weights (4) is assigned in order to judge whether those facts a group can lead the adoption of a fabric defect.

$$
\begin{aligned}
& {\left[b_{1}, \quad b_{2}, \quad b_{3}, \cdots \cdots b_{12}\right] \ldots \ldots \ldots \ldots \ldots \ldots} \\
& {\left[\beta_{1}, \quad \beta_{2}, \quad \beta_{3}, \cdots \cdots \beta_{12}\right] \ldots \ldots \ldots \ldots}
\end{aligned}
$$

The assignment method of $\beta_{j}(j=1,2,3 \ldots \ldots 12)$ in (4) is as follows. Firstly, a term of the same meaning to some fact term $a_{i}(i=1,2,3, \ldots \ldots 12)$ of (1) was searched and then a value was assigned. For example, if $b j$ is the same meaning as $a_{i}$, then $\beta_{j}=\alpha_{i}$. If $a_{i}$ with the same meaning term to some $b_{j}$ does not exist, then $b_{j}$ is assigned by following two ways.

(1) If the relation between $b_{j}$ and the adoption can not be admitted, then $\beta_{j}=0$.

(2) If $b_{j}$ is disadvantageous for adopting a defect, then $\beta_{j}$ will be assigned with minus value. In this way, the absolute value of $\beta_{j}$ will be determined in accordance with how disadvantageous it will be.

In the experiment, if the sum of (4) was over the threshold $\phi A$, then the defect was adopted, or

If $\Sigma \beta_{j} \geqq \phi A$, then the defect was adopted.

Adopted confidence quality was given by $\Sigma \beta_{j} / \Sigma \alpha_{i}$, if all fact terms were satisfied, the confidence quality was 1.0 , if all not, then it would be 0 .

\section{Experiment}

\subsection{Sample}

The sample used in experiment was white glass fabric made by Nittobo, which reflection characteristic was good. In these sample, there were defect lashingin, weft tearing, cloth stain, slack pick, broken pick, slough-off, bonded yarn, nap woven-in, etc.

In order to keep the sample in plane state, the sample on $50 \mathrm{~mm} \times 50 \mathrm{~mm}$ glass plate was pasted by using a double-faced cellotape.

\subsection{Experiment method}

\subsubsection{Basic properties of signal detecting apparatus}

The outline of the signal detection apparatus is shown in Fig. 5. The experimental equipment was composed of He-Ne laser generator SL-102 of which wave length was $0.628 \mu \mathrm{m}$, optical sensor PMT (Photomultiplier Tube) PH-7B, personal computer PC-98VX, rotation table and electronic $X-Y$ stage.

Moreover, an A/D converter AB98-05 was used to transform analog signal to digital signal. Other, to compare the signal effect of reflected light from each direction, we restricted the PMT on a round rail, and detected the signal with the same distance $(21.0 \mathrm{~mm})$ between the irradiated Point and the surface of the 


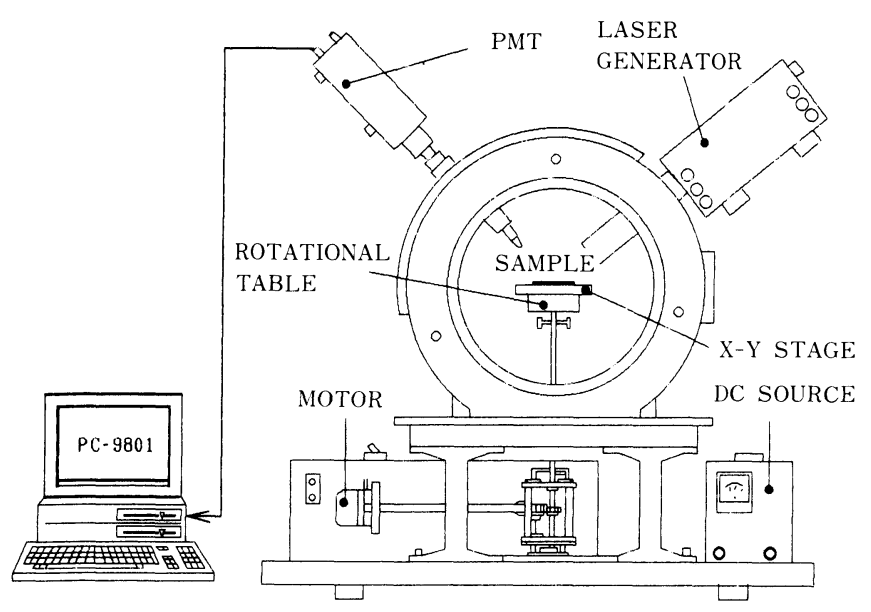

Fig. 5 Apparatus for measurement of the reflected pattern

sensor PMT.

The optical sensor PH-7B made by JEMIC was highly precise, small-sized, light weight type. Photomultiplier tube R453 was used as the detecting part of the sensor.

A red filter R-68 was inserted on the optical path to detect the reflected light signal that only came from the laser generator. Under the above condition, it is confirmed that the influence of the other light is nearly zero.

From the above, we judged that the sensor is suitable to the measurement of the fabric reflected light.

\subsubsection{Pinhole on light path}

The optical theory of the signal measurement is shown in Fig. 6. The laser beam (it's diameter is $2 \mathrm{~mm}$ ) is reflected on the surface of fabric sample, and part of the reflected light goes through the pinhole and the filter, then it is transformed to electric signal by PMT. There is not any optical lens and the length of the pinhole $(35 \mathrm{~mm})$ is comparatively long, so the light entered to PMT becomes a parallel beam with the center line of PMT. Therefore the signal of the light is not from all lighted area, but only from an ellipes area that the major axis

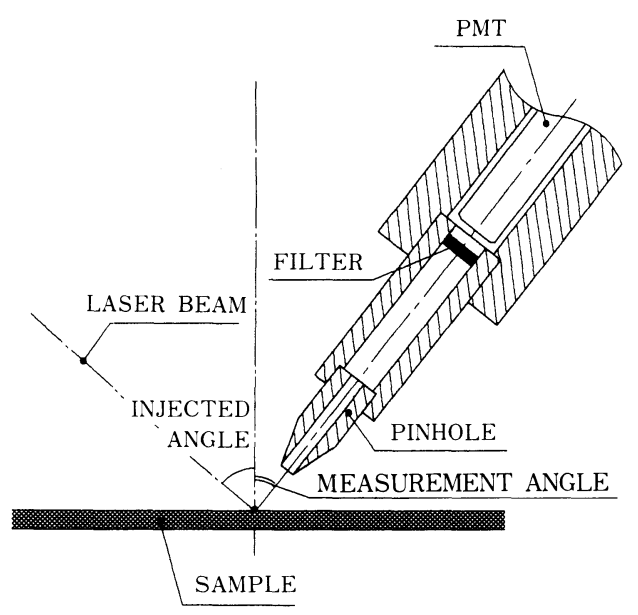

Fig. 6 Schematic diagram of the interior structure of photo-multiplier tube is $b$, the minor axis is $a$, and the minor axis is equal to the diameter of the pinhole. When the diameter of pinhole is $a$, and the reflected light of fabric defect is measured at the angle $\theta$, major axis $b$ is shown as follows.

$$
b=a / \cos \theta
$$

Fig. 7 (a) $\sim$ (f) shows the result taken from experiment about the effect of the pinhole diameter.

As the result analysed before, if the pinhole is too small, the ripple of the noise caused by naps will become large, and the defect discrimination will become difficult. But if the pinhole is too large, the characteristic signal of the fabric will be averaged, in results, the signal becomes small, and the reflection pattern becomes to a circle, and the recognization of fabric defect will become impossible. In this experiment, when the diameter of the pinhole is about $0.7 \mathrm{~mm}$, the ripple of the noise become

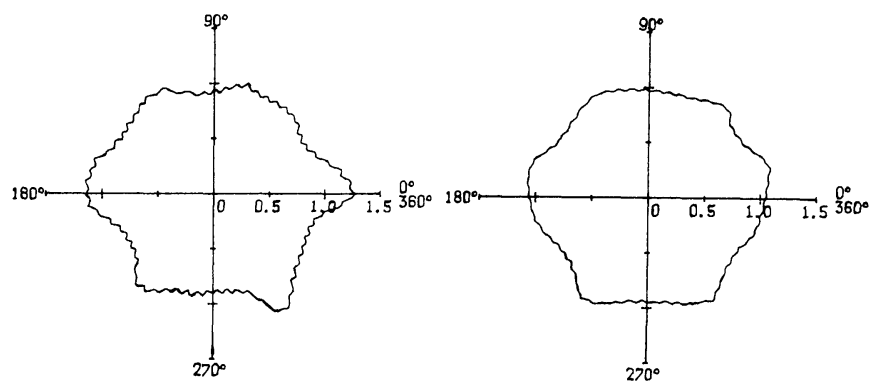

(a)

(b)

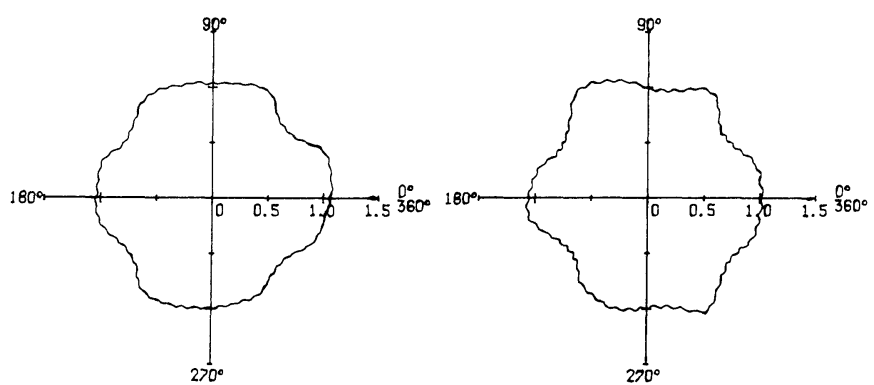

(c)

(d)

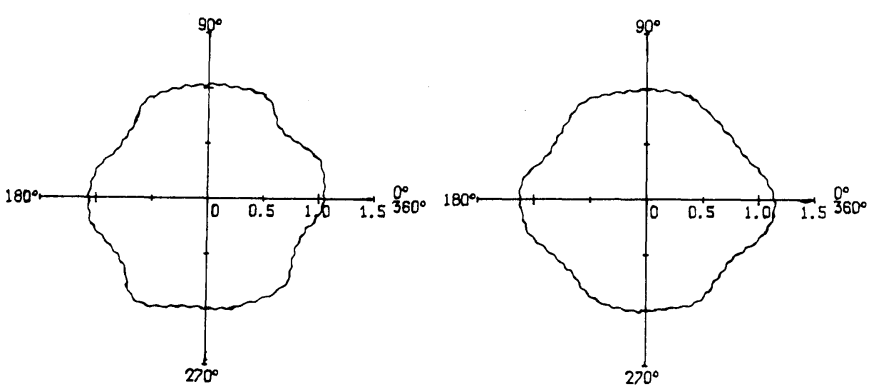

(e)

Fig. 7 Six reflected patterns detected with different pinhole. The incidence angle of laser light is 75 degrees. The measurement angle is 65 degrees. Rotation table takes 10.70 seconds for one revolution. The pinhole diameter is as follows
(a): $0.3 \mathrm{~mm}$
(b): $0.5 \mathrm{~mm}$
(c): $0.7 \mathrm{~mm}$
(d): $1.0 \mathrm{~mm}$
(e): $1.5 \mathrm{~mm}$
(f): $2.0 \mathrm{~mm}$ 

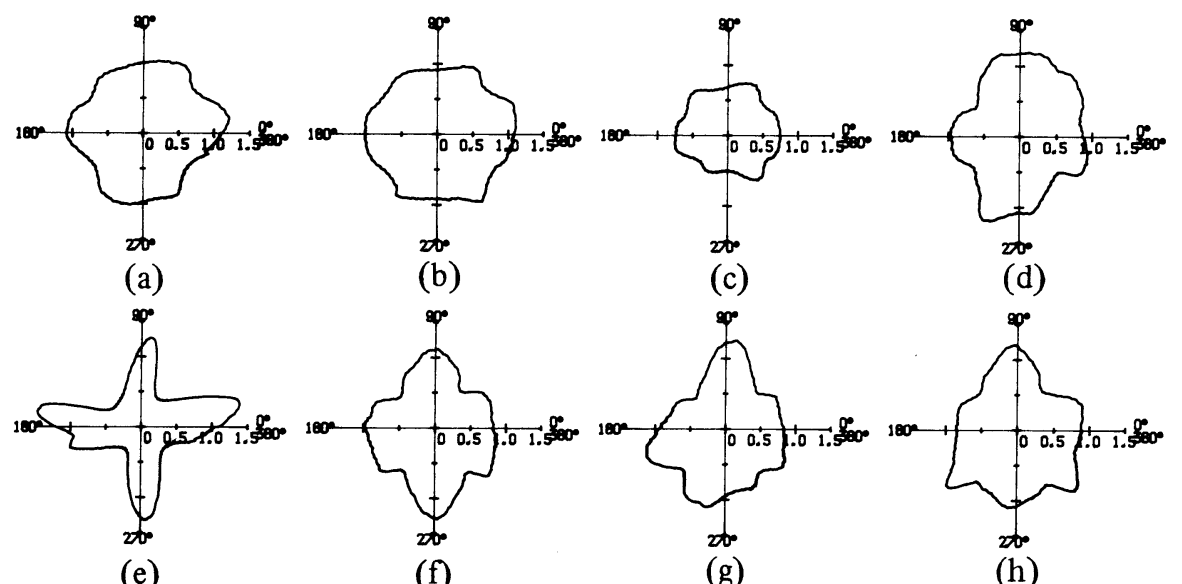

(e)

(f)

(g)

(h)

Fig. 8 Corresponding reflected patterns to the sample of lashing-in, weft tearing, cloth stain, slack pick, broken pick, slough-off, bonded yarn, nap woven-in

small, and the signal of fabric defect will be easy to recognize.

\subsection{Measurement of signal}

In order to create a reflected pattern, the laser beam was shined on the fabric surface, then analogously the reflected light was measured using PMT with rotating the sample and these signals were inputted into the computer after digital transformation. Since the reflected light signal was measured over 360 degrees, in the end, the same information would be got, as it was measured with a fixed incident angle of the laser. Further more, the parameters and the dummy variables were calculated to reason the kind of defect.

Here the incident angle of laser was 75 degrees, the measuring angle of laser was 65 degrees, the rotation speed of the rotation table was $5.61(\mathrm{rpm})$.

\section{Result}

Fig. 8 (a) $\sim($ h) shows the reflected patterns which were detected from the sample lash-in, weft tearing, cloth stain, slack pick, broken pick, slough-off, bonded yarn, nap woven-in. As shown in Fig. 8, it is clear that the reflection pattern at the defect was distorted from normal, and the distortion was dependent on the kind of defect, if an appropriate incident angle of the laser and measuring angle were used. Table 2 shows the parameters. If measured value were calculated due to the upper and lower limits shown in Table 1, the dummy variables shown in Table 3 was taken, then if these values were inputted into the expert system, the defect could be reasoned.

Now, let us see a reasoning process for a broken pick defect. All the conditions (parameters) for recognizing broken pick are as follows.

$$
\left[L(1), D_{a}(1), I_{0}(1), \quad H(1), S_{h}(1), J_{m}(1)\right] \ldots \ldots \ldots \text { (a) }
$$

The numbers in the parentheses are the values of the parameter. Applying following weight (b) to the above term (a).

Table 2 Characteristic parameters

\begin{tabular}{l|c|c|c|c|c|c|c|c|c|c|c|c}
\hline \multicolumn{1}{c|}{ Sample } & $L$ & $L_{s}$ & $D_{p}$ & $D_{i}$ & $D_{a}$ & $I_{0}$ & $R_{a}$ & $H$ & $S_{n}$ & $S_{h}$ & $H_{d}$ & $J_{m}$ \\
\hline Normal & 7.11 & 2.07 & .108 & .925 & 1.29 & 1.98 & 1.25 & 1.04 & 1.25 & .225 & .748 & .284 \\
\hline Lashing-in & 7.40 & 2.07 & .048 & .871 & 1.41 & 2.26 & 1.50 & 1.05 & 2.77 & .317 & .867 & .329 \\
\hline Weft tearing & 6.62 & 2.14 & .062 & .926 & 1.09 & 1.40 & .999 & .997 & 0.23 & .124 & .287 & .189 \\
\hline Cloth stain & 4.71 & 3.43 & .060 & .551 & .784 & .316 & .916 & .656 & 0.60 & .146 & .570 & .443 \\
\hline Slack pick & 6.94 & 2.17 & .125 & .831 & 1.18 & 1.74 & 1.14 & 1.00 & 1.76 & .243 & .541 & .400 \\
\hline Broken pick & 8.66 & 2.11 & .079 & .668 & 1.52 & 3.08 & 1.11 & 1.12 & 4.89 & .746 & .794 & .587 \\
\hline Slough-off & 6.22 & 2.31 & .054 & .760 & 1.07 & 1.21 & .929 & .920 & 1.05 & .140 & .458 & .349 \\
\hline Bonded yarn & 6.57 & 2.47 & .068 & .635 & 1.23 & 1.28 & 1.30 & .903 & 3.22 & .330 & .986 & .535 \\
\hline Nap woven-in & 6.82 & 2.31 & .080 & .717 & 1.37 & 1.63 & 1.52 & .951 & 3.75 & .327 & 1.24 & .449 \\
\hline
\end{tabular}


Table 3 Dummy variables

\begin{tabular}{l|r|r|r|r|r|r|r|r|r|r|r|r}
\hline \multicolumn{1}{c|}{ Sample } & $L$ & $L_{s}$ & $D_{\rho}$ & $D_{i}$ & $D_{a}$ & $I_{0}$ & $R_{a}$ & $H$ & $S_{n}$ & $S_{h}$ & $H_{d}$ & $J_{m}$ \\
\hline Normal & 2 & 2 & 2 & 2 & 2 & 2 & 2 & 2 & 2 & 3 & 2 & 2 \\
\hline Lashing-in & 2 & 2 & 2 & 2 & 1 & 2 & 1 & 2 & 1 & 1 & 1 & 2 \\
\hline Weft tearing & 3 & 2 & 2 & 2 & 3 & 3 & 3 & 2 & 3 & 3 & 3 & 3 \\
\hline Cloth stain & 3 & 1 & 2 & 3 & 3 & 3 & 3 & 3 & 2 & 3 & 2 & 1 \\
\hline Slack pick & 2 & 2 & 1 & 3 & 3 & 2 & 2 & 2 & 2 & 2 & 2 & 1 \\
\hline Broken pick & 1 & 2 & 2 & 3 & 1 & 1 & 2 & 1 & 1 & 1 & 2 & 1 \\
\hline Slough-off & 3 & 1 & 2 & 3 & 3 & 3 & 3 & 3 & 2 & 3 & 2 & 2 \\
\hline Bonden yarn & 3 & 1 & 2 & 3 & 2 & 3 & 1 & 3 & 1 & 1 & 1 & 1 \\
\hline Nap woven-in & 3 & 1 & 2 & 3 & 1 & 3 & 1 & 3 & 1 & 1 & 1 & 1 \\
\hline
\end{tabular}

$\left[L(6), D_{a}(4), I_{o}(5), H(5), S_{n}(3), J_{m}(2)\right] \ldots \ldots \ldots$ (b)

There are twelve terms in (b), but there are only six terms in (a), because the other terms are not admitted for the relation to adopt the broken pick, in other word, the weight is zero and they are omitted.

These weights (b) represent how much the fact terms contribution to the reason to adopt the broken pick. The fact terms taken from experiment are as follows.

$$
\begin{aligned}
& {\left[L(1), L_{s}(2), D_{p}(2), D_{i}(3), D_{a}(1), I_{o}(1)\right.} \\
& \left.R_{a}(2), H(1), S_{n}(1), S_{n}(1), H_{d}(2), J_{m}(1)\right] \ldots \ldots \ldots
\end{aligned}
$$

In the above terms, there are six facts $L, D_{a}, I_{0}, H$, $S_{h}, J_{m}$, which have the same meaning with the facts (a) for recognizing the broken pick. The other facts do not exist at (a) and the weight is assigned to zero. In the end, following weights are assigned.

$$
\left[L(6), D_{a}(4), I_{0}(5), H(5), S_{h}(3), J_{m}(2)\right] \ldots \ldots \ldots
$$

The sum of the weights becomes 25 , as it is larger than the threshold 20, so broken pick is adopted. The sum of (d) is equal to the sum of (b), then adopted confidencial quality is 1.0 .

The reasoning process in detail will be performed as follows. The other defects will not be adopted. For example, in the case of woven bar, the facts are as follows.

$$
\left[L(2), L_{s}(1), D_{i}(3), D_{a}(1), H(3), S_{n}(1) H_{t}(1), J_{m}(2)\right]
$$

Weights (f) will be applied to the above terms.

$$
\left[L(2), L_{s}(3), D_{i}(1), D_{a}(3), H(3), S_{h}(3), H_{d}(3), J_{m}(3)\right]
$$

These weights represent how much (e) contributed to the reason in order to adopt the woven bar. The facts taken from the experiment was as same as (c). There were three facts $D_{i}, D_{a}, S_{h}$, which had the same meaning with the facts (e) for recognizing the woven bar.

The other terms did not exist at (e), and the weight was assigned to zero. In the end following weights were assigned.

$\left(D_{i}(1), \quad D_{a}(3), \quad S_{h}(3)\right]$

The sum of the weights was seven, less than the threshold 18 , so the woven bar was not adopted.

In this experiment, the recognization rate about 70 95 percentage was taken.

\section{Conclusion}

1) The reflected pattern of fabric was dependent on the structure of fabric. The reflected pattern at the defect was distorted from normal and that distortion was dependent on the kind of defect.

2) In order to detect the signal of the light only from laser, a pinhole was set on the optical path, and when the diameter of the pinhole was about $0.7 \mathrm{~mm}$, the ripple of the noise was small, and the signal of the fabric defect would be easy to detect.

3) Twelve parameters were introduced as the characteristic of the reflected pattern and by using these parameters, the reflected patterns were discriminated with them.

4) Table reasoning method was introduced to recognize the fabric defect and the method was suitable for processing the fuzzy information such as reflected pattern.

\section{Reference}

[1] K.Furuta, E.Kuze; Sen-i Gakkaishi, 29, T224 (1973)

[2] R.Akiyama, E.Kuze; Sen-i Gakkaishi, 35, T202 (1978)

[3] E,Kuze, T.Nishide; Sen-i Gakkaishi, 32, T178 (1975)

[4] G.J.Agin; An Experimental Vision System for Industrial Application, proc. 5-th international symposium on industrial robots, pp.135/139, 1975.

[5] S.S.Shigeru; TELL Users Manual, p.6-45, 1986 